\title{
Inhibition of Kelch-like epichlorohydrin-related protein 1 promotes the progression and drug resistance of lung adenocarcinoma
}

\author{
Hong Gao ${ }^{\text {Equal first author, } 1}$, Peipei Tang ${ }^{\text {Equal first author, } 2}$, Kejie Ni ${ }^{1}$, Lun Zhu ${ }^{3}$, Song Chen ${ }^{2}$, Yulong Zheng ${ }^{\text {Corresp., } 1}$, Yufeng Wan \\ Corresp. 1 \\ ${ }^{1}$ Department of Respiratory Diseases, The Affiliated Huai'an Hospital of Xuzhou Medical University, Huai'an, Jiangsu, China \\ 2 Institute of Medicinal Biotechnology, Jiangsu College of Nursing, Huai'an, Jiangsu, China \\ 3 Department of Pathology, The Affiliated Huai'an Hospital of Xuzhou Medical University, Huai'an, Jiangsu, China \\ Corresponding Authors: Yulong Zheng, Yufeng Wan \\ Email address: ha183@163.com, ggwanyufeng@163.com
}

Background: Lung cancer is a common malignant carcinoma of respiratory system with high morbidity and mortality. Kelch-like epichlorohydrin-related protein 1 (Keap1), a member of the BTB-Kelch protein family, has been reported as an important molecule in several cancers. However, its potential role in tumor is still controversial. Here we aim to clarify the effect of Kelch-like epichlorohydrin-related protein 1 (Keap1) on the biological characteristics and chemotherapy resistance in lung adenocarcinoma.Methods: Immunohistochemistry was conducted to compare Keapl expression in lung adenocarcinoma (LUAD) tissues and matched non-cancerous tissues, and the correlation between Keap1 expression and clinicopathological features was analyzed. Subsequently, the stable A549 and H1299 cell lines with Keap1 knockdown or overexpression were constructed using lentivirus. The roles of Keapl on the cell proliferation, migration, invasion and drug resistance were investigated by colony formation assay, cell proliferation assay, wound scratch test, transwell invasion assay and drug sensitivity assay, respectively. Results: Keap1 was lowly expressed in tumor tissues compared to matched non-cancerous tissues, and its expression was correlated with TNM stage and lymph node metastasis. Early stage (I) tumors without lymph node metastasis had higher levels of Keap1 expression compared with late-stage tumors (II, III) with the presence of lymphatic metastasis. Colony formation assays showed that Keap1 knockdown promoted the proliferation of A549 and H1299 cells, and the cell growth curves further confirmed this feature. In contrast, wound scratch and transwell invasion experiments showed that Keap1 overexpression inhibited cell migration and invasive malignancy. The IC50 for cisplatin and paclitaxel were significantly increased by Keap1 knockdown in A549 and H1299 cell lines.Conclusion: Keap1 knockdown promotes tumor cell growth, proliferation, invasion, 
metastasis and chemotherapy resistance in lung adenocarcinoma. It may be a potential tumor marker to guide the staging and treatment of lung cancer. 
1 Inhibition of Kelch-like epichlorohydrin-related protein 1 promotes the progression and drug 2 resistance of lung adenocarcinoma

3 Hong Gao ${ }^{1 *}$, Peipei Tang ${ }^{2 *}$, Kejie Ni ${ }^{1}$, Lun $\mathrm{Zhu}^{3}$, Song Chen ${ }^{2}$, Yulong Zheng ${ }^{1}$ and Yufeng Wan ${ }^{1}$

$4{ }^{1}$ Department of Respiratory Diseases, The Affiliated Huai'an Hospital of Xuzhou Medical University,

5 Huai'an, Jiangsu, China

6 '2Institute of Medicinal Biotechnology, Jiangsu College of Nursing, Huai'an, Jiangsu, China

$7{ }^{3}$ Department of Pathology, The Affiliated Huai'an Hospital of Xuzhou Medical University, Huai'an,

8 Jiangsu, China

$9 *$ These authors contributed equally to this work.

10 Corresponding Authors:

11 Yulong Zheng (ha183@163.com), Yufeng Wan (ggwanyufeng@163.com) 


\section{Abstract}

13 Background: Lung cancer is a common malignant carcinoma of respiratory system with high morbidity

14 and mortality. Kelch-like epichlorohydrin-related protein 1 (Keap1), a member of the BTB-Kelch protein

15 family, has been reported as an important molecule in several cancers. However, its potential role in tumor

16 is still controversial. Here we aim to clarify the effect of Kelch-like epichlorohydrin-related protein 1

17 (Keap1) on the biological characteristics and chemotherapy resistance in lung adenocarcinoma.

18 Methods: Immunohistochemistry was conducted to compare Keap1 expression in lung adenocarcinoma

19 (LUAD) tissues and matched non-cancerous tissues, and the correlation between Keap1 expression and

20 clinicopathological features was analyzed. Subsequently, the stable A549 and H1299 cell lines with Keap1

21 knockdown or overexpression were constructed using lentivirus. The roles of Keap1 on the cell

22 proliferation, migration, invasion and drug resistance were investigated by colony formation assay, cell

23 proliferation assay, wound scratch test, transwell invasion assay and drug sensitivity assay, respectively.

24 Results: Keap1 was lowly expressed in tumor tissues compared to matched non-cancerous tissues, and its expression was correlated with TNM stage and lymph node metastasis. Early stage (I) tumors without lymph node metastasis had higher levels of Keap1 expression compared with late-stage tumors (II, III) with

27 the presence of lymphatic metastasis. Colony formation assays showed that Keap1 knockdown promoted the proliferation of A549 and H1299 cells, and the cell growth curves further confirmed this feature. In contrast, wound scratch and transwell invasion experiments showed that Keap1 overexpression inhibited cell migration and invasive malignancy. The IC50 for cisplatin and paclitaxel were significantly increased by Keap1 knockdown in A549 and H1299 cell lines.

32 Conclusion: Keap1 knockdown promotes tumor cell growth, proliferation, invasion, metastasis and

33 chemotherapy resistance in lung adenocarcinoma. It may be a potential tumor marker to guide the staging 34 and treatment of lung cancer. 


\section{Introduction}

As the world's largest cancer killer, lung cancer accounts for approximately 2.09 million new cases (11.6\% of all new cancer cases) and 1.77 million lung cancer-related deaths (18.4\% of all cancer deaths) each year. Its mortality and incidence rates rank first among all cancers ${ }^{1}$. LUAD is the most common form of non-small-cell lung cancer, and mostly at advanced stage when diagnosed ${ }^{2}$. Keap1 protein belongs to the BTB-Kelch protein family. The BTB-Kelch protein family mainly assembles with Cullin3 and Rbx1 to form multi-subunit Cullin-RING ligases (CRLs) for protein ubiquitination ${ }^{3}$. Keap1 comprises five domains: - N-terminal region (NTR), Broad complex, Tramtrack and Bric-à-Brac (BTB), intervening region (IVR), double glycine repeat (DGR) and C-terminal region (CTR). The BTB region acts as a substrate-specific adaptor for Cullin3 ubiquitin ligase, which mediates the homodimerization of Keap1 and the subsequent constitutive ubiquitination of Nrf2. The IVR region contains cysteine residues which are sensitive to oxidation, or to covalent modification by electrophiles. The DGR and CTR regions are also called DC domains, mainly mediating the interaction with and $\mathrm{Nrf} 2^{4,5}$.

The most important function of Keap1 in the body is to play an anti-oxidative stress effect by the Keap1-nuclear factor E2-related factor2 (Nrf2) pathway. Under steady-state conditions, Keap1 binds to Nrf2 and anchors it in the cytoplasm. At the same time, it assembles with the Cullin3 protein to form a Cullin-RING E3 ligase complex for the degradation of Nrf2 by the 26S proteasome, so that Nrf2 is in a non-free and continuously degraded non-free state ${ }^{5}$. Keap1 protein contains a total of 27 cysteine residues (Cys), which are vulnerable to reactive oxygen species (ROS), or to covalent modification by electrophiles, especially the Cys151, Cys257, Cys273, Cys288, Cys297, Cys434 and Cys66 ${ }^{6}$.This characteristic determines the high redox sensitivity of Keap1. Under conditions of oxidative stress, or the presence of electrophilic xenobiotics, Keap1 cysteine residues are oxidized, thereby altering the Cullin-RING E3 ligase complex and losing the Keap1-Nrf2 interaction, ultimately leading to Nrf2 translocation and nuclear accumulation. In nucleus, the actived Nrf2 forms a heterodimer with the small Maf (sMAF) proteins, which belongs to Maf family of transcription factors ${ }^{7}$. The heterodimer recognizes antioxidant response element (ARE) that regulates the downstream transcription of target genes, which encode proteins acting as redox balancing 
61 factors, detoxifying enzymes, stress response proteins and metabolic enzyme, thereby exerting 62 cytoprotective effects ${ }^{8}$.

63 However, as one of the main factors regulating oxidative stress in the body, various recent studies have

64 shown that Keap1 mutations are associated with cancers. Schulze et al. found Keap1 mutations in about

$6514 \%$ of hepatocellular carcinomas by exon sequencing ${ }^{9}$. Chu XY et al. reported Keap1 heterozygous

66 missense mutations in cervical cancer ${ }^{10}$. Besides, Keap1 mutations have also been reported in lung cancer.

67 An earlier study reported a mutation rate of Keap1 in lung squamous cell carcinoma is about $12 \%{ }^{11}$. While

68 recently, a large-scale genomic exon sequencing study including 660 cases of lung adenocarcinoma and

69484 cases of lung squamous carcinoma found that Keap1 gene was significantly mutated only in lung

70 adenocarcinoma $^{12}$. However, Keap1 expression in solid tumors is variable and correlate with

71 clinicopathological features. Researchers found that Keap1 protein was highly expressed in both

72 endometrial and pancreatic cancers by immunohistochemistry, and that high levels of Keap1 protein

73 expression were associated with poor prognosis in endometrial cancer ${ }^{13,14}$. While another investigators

74 reported that Keap1 protein was lowly expressed in breast cancer and osteosarcoma tissues and correlated

75 with clinicopathological features such as lymph node metastasis ${ }^{15,16}$. These studies suggest that the

76 abnormal status of Keap1 in the body may influence tumorigenesis and progression.

77 So, in this study, we aimed to identify the effect of Keap1 on the biological characteristics and 78 chemoresistance in LUAD, and to provide a new entry point for clinical diagnosis and targeted therapy. 


\section{Materials \& Methods}

\section{Patient samples}

The Institutional Review Board of the Affiliated Huai'an Hospital of Xuzhou Medical University

82 (HEYLL201920) approved this study and all subjects signed an informed consent form prior to the study.

83 Lung cancer specimens for this study were collected retrospectively from 37 patients in the hospital from

84 January 2016 to December 2018. Matched non-cancerous tissue was defined as $>5-10 \mathrm{~cm}$ from the

85 cancerous tissue. The inclusion criteria were as follows: 1) Pathologically confirmed adenocarcinoma of

86 the lung according to the "ICD-O-3 Hist / Behavior"; 2) All patients underwent radical resection without

87 induction of chemotherapy or radiation therapy prior to surgery.3) Patients did not have other malignancies

88 prior to diagnosis; 4) Re-staging TNM staging according to the American Joint Committee on Cancer 8th

89 edition TNM staging based on original staging and tumor size provided ${ }^{17}$; The exclusion criteria were as

90 follows: 1) Patients with comorbid acute cardiovascular and autoimmune diseases; 2) Patients with missing

91 or unknown clinical information.

\section{Immunohistochemistry (IHC) assay}

The Immunohistochemistry was carried out according to standard protocol. In shortly, all paraffin specimens were sliced to a thickness of $4 \mu \mathrm{m}$, then deparaffinized and hydrated and heated in Tris/EDTA buffer $\mathrm{pH} 9.0$ for antigen retrieval. The Keap1 antibody (1:500, ab227828, Abcam) was incubated overnight at $4{ }^{\circ} \mathrm{C}$ and the specimens were visualized with a light microscope (Olympus, Japan). Two pathologists completed the readings independently. The average number of positive cells in each field was analyzed as the percentage of positive cells in the section and scored using the Fromowitz criteria ${ }^{18}: 0 \%-5 \%$ was scored as $0,6 \%-25 \%$ was scored as $1,25 \%-50 \%$ was scored as $2,51 \%-75 \%$ was scored as 3 , and $>75 \%$ was scored as 4 . The intensity of staining was scored according to the staining characteristics of most positive cells: 0 score for no staining, 1 score for light yellow, 2 score for brown, and 3 score for dark brown. The final score was derived by summing the percentage of stained cells and the corresponding intensity, with $0-1$ 
104 strongly positive $(+++)$. Where $(-)$ and $(+)$ recorded as low expression, $(++)$ and $(+++)$ recorded as high 105 expression groups.

\section{Cell culture and transfection}

107 The human bronchial epithelial HBE cell line, human lung cancer cell lines NCI-A549, NCI-H1299, 108 NCI-H460, NCI-H2126 and human embryonic kidney HEK-293FT cell lines were obtained from Huai'an 109 Key Laboratory of Gastrointestinal Cancer. The HBE, NCI-H460 and NCI-H2126 cell lines were cultured 110 in RPMI 1640 medium (Gibco, USA) with 10\% fetal bovine serum (FBS, Gibco, USA) and incubated in a 111 moist air incubator with $5 \% \mathrm{CO}_{2}$ at $37{ }^{\circ} \mathrm{C}$. The NCI-A549, NCI-H1299 and HEK-293FT cell lines were 112 maintained in Dulbecco's Modified Eagle's Medium (DMEM, Gibco, USA) containing 10\% FBS in 5\% $113 \mathrm{CO}_{2}$ at $37^{\circ} \mathrm{C}$. Fresh medium was replaced every 1-2 days. The cells were passaged in a dilution ratio of 1:4 114 approximately every two days when the confluency reached about $80 \%$. The cDNA sequence of Keap1 115 gene (GenBank accession No. NM_203500) was cloned into Lenti-EFs-Flag-puro vector for keap1 116 overexpression. Short hairpin RNA (shRNA) sequences targeting Keap1 was constructed into pLKO.1

117 lentiviral vector for keap1 knockdown. For lentiviral packaging system, we incubated HEK-293FT cells in 118 six-well plate and when the cell density reached $90 \%$ or higher, the VSVG envelope plasmid, Gag/Pol 119 packaging plasmid, Keap1 overexpression plasmid, Keap1 knockdown plasmid and the control plasmid 120 were transfected into HEK-293FT cells according to the Lipo8000 ${ }^{\mathrm{TM}}$ transfection kit (Beyotime 121 Biotechnology, China). The virus particles were collected after 48 hours. For construction of stable cell 122 lines, the A549 and H1299 cell lines were seeded in 24-well plate and when fusion reached nearly 30\%, 123 appropriate amounts of virus were added. After 24 hours, we replaced the fresh medium, waited for the cell 124 density to reach approximately $80 \%$, and then added $2 \mathrm{ug} / \mathrm{ml}$ puromycin (Sigma, USA) to screen the stable 125 cell lines.

\section{Western blot analysis}

127 Protein lysates containing DL-Dithiothreitol (DDT) and Sodium dodecyl sulfate (SDS) were used to 128 extract total proteins. The cytosolic and nuclear fractions of Nrf2 were prepared using a Nuclear and 
129 Cytoplasmic Protein Extraction Kit (Beyotime Biotechnology, China). Protein quantification was

130 performed using BCA protein quantification kit (Beyotime Biotechnology, China). The volume that 131 contains $20 \mu \mathrm{g}$ protein was calculated to be taken as samples for $8 \%-13 \%$ sodium dodecyl sulfate 132 polyacrylamide gel electrophoresis (SDS-PAGE). Then the gel was transferred to a nitrocellulose (NC) 133 membrane with constant voltage at $30 \mathrm{~V}$ in an ice-water bath for overnight. After transferring the 134 membrane, it was blocked with 5\% nonfat-dried milk for $1 \mathrm{~h}$ at room temperature. The NC membrane was 135 then incubated with primary antibody for $4 \mathrm{~h}$ at $37^{\circ} \mathrm{C}$ and the appropriate HRP-conjugated secondary 136 antibody was further incubated for $1 \mathrm{~h}$ at room temperature. The Enhanced chemiluminescence (Beyotime 137 Biotechnology, China) reagents $\mathrm{A}$ and $\mathrm{B}$ were mixed thoroughly in a 1:1 ratio, incubated with NC 138 membrane for $1 \mathrm{~min}$, and then exposed the $\mathrm{NC}$ membrane to the dark chamber of a chemiluminescence 139 imaging system (Azure US Biosystems). $\beta$-actin was as an internal control. Image $\mathbf{J}$ software was applied 140 for the quantification of protein. The primary antibodies used in western blotting were listed in Table 1.

\section{Reverse transcription-quantitative polymerase chain reaction (RT-qPCR)}

142 Total RNA was extracted using Trizol reagent (Invitrogen, USA). The integrity of RNA was tested by 143 agarose gel electrophoresis and competent RNA produced clear 28S and 18S rRNA bands after processing.

144 The quality of RNA was tested using a spectrophotometer/fluorometer (DeNovix, USA), only RNA 145 samples with a 260/280 ratio between 1.8-2.2 were used in reverse transcription to synthesize cDNA using 146 the PrimeScript ${ }^{\mathrm{TM}}$ RT reagent Kit with gDNA Eraser reverse transcription kit (Takara, Japan). Then, the 147 fluorescent quantitative PCR reaction system was analyzed for $100 \mathrm{ng}$ of cDNA template following the 148 protocol of TBGreen ${ }^{\circledR P r e m i x}$ Ex Taq ${ }^{\mathrm{TM}}$ kit (Takara, Japan). The specific PCR amplification procedure was 149 as follows: $95^{\circ} \mathrm{C}$ for 30 seconds, then $95^{\circ} \mathrm{C}$ for 5 seconds, $60^{\circ} \mathrm{C}$ for 30 seconds with 40 cycle. The 3150 glyceraldehyde-phosphate dehydrogenase (GAPDH) was applied as an endogenous reference. Relative 151 quantitative analysis using $2^{-\Delta \Delta \mathrm{Ct}}$ method. The primer sequences were as following: GAPDH-F: 5'-ACT 152 TTG GTA TCG TGG AAG GAC TC-3', GAPDH-R: 5'GAG GCA GGG ATG ATG TTC TGG-3; Keap1- 
154 CGC AGC GC-3'.

\section{Colony formation assay}

156 Cells in logarithmic growth phase were inoculated 500-1000 cells per well in six-well plates, and 157 maintained at $37^{\circ} \mathrm{C}$ in an atmosphere of humidified air with $5 \% \mathrm{CO}_{2}$. The medium was replaced with fresh 158 medium every 5 days. After ten days later, the six-well plates were taken out and the medium were removed.

159 And the cells were fixed with $4 \%$ paraformaldehyde for $30 \mathrm{~min}$, followed by staining with crystal violet for $16010 \mathrm{~min}$, then rinsed slowly and air-dried. Pictures were taken in the darkroom of Gel luminescence Imaging 161 System (Azure Biosystems, USA), and the cell colony numbers were counted using Image-Pro Plus 162 software.

\section{Wound scratch test}

Cells in logarithmic growth phase were spread in six-well plates at a density of $1 \times 10^{6}$ cells per well.

165

166

167

168

169

170

171

172

173

174

175

176

177

When the cell confluence reached $95 \%-100 \%$, a 200 ul yellow pipette tip was used to draw three vertical lines in each well and the exfoliated cells were washed away with PBS. Then, $2 \mathrm{ml}$ of DMEM medium without FBS was added to each well and further incubated in a $37^{\circ} \mathrm{C}, 5 \% \mathrm{CO} 2$ incubator. Under the orthomosaic microscope (Olympus, Japan), the changes of the scratch width were observed and photographed $(\times 40)$ at a certain time. The Image $J$ software was used to quantitative analysis for the scratch area. Cell migration rate $(\%)=($ initial scratch area - scratch area at specified time) $/$ initial scratch area $\times$ $100 \%$.

\section{Transwell invasion assay}

Transwell invasion assay was performed as previously described ${ }^{19}$. In brief, an appropriate number of cells were seeded in the upper chamber of the 24-well Transwell chamber (Corning, USA), which containing 200ul serum-free medium. $600 \mu \mathrm{l}$ of DMEM containing 20\% FBS was plated in the bottom chamber. Following a $20 \mathrm{~h}$ incubation in $5 \% \mathrm{CO}_{2}$ at $37^{\circ} \mathrm{C}$, the cells were rinsed, fixed with pre-cooled methanol for 30 minutes and stained with crystal violet for 15 minutes. The cells on the upper-side of the 
178 membrane were then removed with a clean cotton swab, and the cells on the under-side were observed and

179 photographed under a light microscope (Olympus, Japan). The number of invaded cells was counted in

180 three randomly selected fields using the Image-Pro plus software.

\section{Cell proliferation assay}

$1821 \times 10^{5}$ cells were seeded at 24-well plate. Each group of cells repeated in three parallel multiple wells.

183 Then the cells were in culture medium at $37^{\circ} \mathrm{C}$ in a $5 \% \mathrm{CO}_{2}$ incubator, and digested with $0.25 \%$ trypsin

184 (Gibco, USA) after 24h, 48h, 72h and 96h, respectively. The digested cells were then counted with Count 185 star automated cell counter (AlitLifeScience, China) and cell survival curves were plotted.

\section{Drug sensitivity assay}

187 The anti-tumor drug resistance on cells was measured by cell viability using the Cell Counting Kit-8 188 solution (CCK-8, MedChemExpress, USA) as previously described ${ }^{20}$. Briefly, cells were seeded in 96-well 189 plates at $5 \times 10^{\wedge} 3$ cells/well in complete medium and incubated overnight. Then varying concentrations of 190 cisplatin and paclitaxel (Qilu Pharmacy Co. Ltd. China) were added into the medium and incubated for 24$19148 \mathrm{~h}$ and 10ul CCK-8 was added to each well for another $1 \mathrm{~h}$. Triplicate wells were analyzed for each 192 concentration. The absorbance was measured at $450 \mathrm{~nm}$ under a microplate reader (Bio-Rad, UAS). Cell 193 survival rate $(\%)=[(\mathrm{As}-\mathrm{Ab}) /(\mathrm{Ac}-\mathrm{Ab})] \times 100, \mathrm{As}=$ absorbance of experimental wells (absorbance of wells 194 containing cells, culture medium, CCK-8 and treated with different concentrations of cisplatin), Ab = 195 absorbance of blank wells (absorbance of wells containing medium and CCK-8), Ac $=$ absorbance of 196 control wells (absorbance of wells containing cells, medium and CCK-8). The half-maximal inhibitory 197 concentration (IC50) was calculated from the rate of cell survival after normalization by the probit 198 transformation.

\section{Establishment of model of oxidative stress induced by $\mathbf{H 2 O 2}$}

200 As previously described ${ }^{21}, \mathrm{H} 2 \mathrm{O} 2$ (Sigma, USA) was used to the establishment of a model of oxidative 201 stress. In brief, the lung cancer cell lines, A549 cell lines with different levels of Keap1 were pre-treated 202 with $200 \mu \mathrm{M} \mathrm{H} 2 \mathrm{O} 2$ for 30 min and H1299 cell lines with different levels of Keap1 were pre-treated with $203100 \mu \mathrm{M}$ H2O2 for 30 min respectively. 


\section{Statistical analysis}

205 SPSS software (version 26.0, USA) and GraphPad Prism software (version 8.0.2, CA) were used for 206 statistical analysis. The measurement data were expressed as mean \pm standard deviation $(\mathrm{x} \pm \mathrm{SD})$, and 207 unpaired t-test was used for comparison between groups. Count data were expressed as (\%), and Fisher's 208 exact test was used to compare the clinical data and immunohistochemical expression. all statistical tests 209 were two-sided probability tests. $\mathrm{P}<0.05$ values were considered significant. 


\section{Results}

211 Keap1 was low expressed in lung adenocarcinoma (LUAD) tissue and cell lines.

212 Thirty-seven cases of LUAD and matched non-cancerous tissues were detected by IHC assays. The

213 result showed that Keap1 protein was mainly distributed in the cytoplasm, and rarely in nuclei (Fig. 1A,

214 1B). The extent of Keap1 expression was significantly higher in para-cancerous tissues than in LUAD

215 tissue. Statistical analysis demonstrated that Keap1 was highly expressed in $67.6 \%$ of paraneoplastic tissues 216 compared to $37.8 \%$ of LUAD tissues, and this difference was statistically significant $(\mathrm{p}=0.019)$. (Table 2).

217 Next, we further detected the expression levels of Keap1 protein in human lung cancer cell lines and human

218 bronchial epithelial HBE cell line by western blot analysis and founded that lower expression of Keap1 in

219 all cancer cell lines compared to HBE (Fig. 1C, 1D). More importantly, the fold change of Keap1 mRNA 220 was significantly lower than that of HBE by RT-qPCR (Fig. 1E).

221

222

223

224

225

226

227

228

229

230

231

232

233

234

235

\section{Keap1 low expression was mainly presented in advanced stage in LUAD tumors}

We also analysed the association between Keap1 expression and clinicopathological features in the 37 LUAD patients, and noted that Keap1 high expression were more commonly observed in early stage (I) than in late-stage (II and III) patients $(\mathrm{P}=0.009)$. Moreover, Keap1 expression was negatively correlated with lymph node metastasis, higher levels of Keap1 expression were more likely associate without lymph node metastasis $(\mathrm{P}=0.017)$. But no correlation was observed between the expression levels of Keap1 and any other clinical parameters, including age, gender, smoking history, tumor location, differentiation and tumor max diameter $(\mathrm{P}>0.05)$ (Table 3). Taken together, the above date indicated that down-regulation of Keap1 may be a critical event in tumor progression.

\section{A549 and H1299 cell lines overexpressing and knocking down Keap1 were successfully constructed.}

Due to the low expression of Keap1 in LUAD patients and cell lines, we chose cells with the relatively high or low expression of Keap1 to more effectively investigate the phenotype changes. Then we stably knocked down and overexpressed Keap1 in A549 and H1299 cell lines using a lentiviral packaging system. To determine whether the A549 and H1299 cell lines overexpressing and knocking down Keap1 were successfully constructed, we examined the mRNA and protein expression levels of Keap1 by RT-qPCR

Peer] reviewing PDF | (2020:12:56716:1:1:NEW 31 May 2021) 
236 and western blotting. As expected, compared with the control group, the Keap1 protein expression levels

237 were significantly increased in the A549 and H1299 cell line overexpression group, while decreased in the 238 knockdown group (Fig. 2A, 2B), RT-qPCR further validated the observations in western blotting assay 239 (Fig. 2C), indicating that the Keap1 overexpression and knockdown A549 and H1299 cell lines were 240 successfully constructed.

\section{Keap1 knockdown facilitated A549 and H1299 cell lines proliferation}

242 To identified the impact of keap1 on proliferation, we performed colony forming assay. Representative 243 images revealed that cells with low Keap1 expression had an enhanced ability to form colonies, while cells 244 with increased Keap1 expression levels formed fewer colonies compared to the control group (Fig. 3A). 245 Quantification of the total number of colonies largely confirmed our observation (Fig. 3B). The result 246 indicated that stable knockdown of Keap1 facilitated the proliferation of A549 and H1299 cells in vitro, and this effect could be further confirmed by cell growth curves up to $96 \mathrm{~h}$ (Fig. 3C).

\section{Keap1 knockdown promoted the migration and invasion in A549 and $\mathrm{H1299}$ cell lines}

The effect of keap1 on the migration ability in A549 and H1299 cells was examined by scratch test. decreased in all cell groups, and the migration rate of the scratches were the highest in the Keap1 knockdown group, the second highest in the control group, and the lowest in the overexpression groups in A549 and H1299 cell lines. To investigate the role of keap1 in LUAD cell invasion, transwell cell invasion significantly higher in the knockdown group A549 and H1299 cell lines than in the control group, whereas in the overexpressed group, the results were reversed (Fig. 4C, 4D).

\section{Keap1 knockdown facilitated EMT of A549 and H1299 cell lines.}

To assess the role of Keap1 in tumor EMT, we performed western blot analysis of EMT marker proteins. The results showed that the expression of the epithelial marker E-cadherin was significantly elevated in A549 cells overexpressing Keap1, whereas the expression of mesenchymal markers (Ncadherin, Vimentin) and Snail was lower in cells overexpressing Keap1. In contrast, knockdown of Keap1 
262 resulted in decreased expression of E-cadherin and increased expression of N-cadherin, Vimentin and Snail 263 (Fig. 5A, 5B), which were consistent with that observed in H1299 cells (Fig. 5A, 5C).

\section{Keap1 knockdown would lead to the chemotherapy resistance in A549 and H1299 cell lines}

265 To explore the keap1 role on chemoresistance, we treated A549 and H1299 cells lines with different 266 concentrations of cisplatin and paclitaxel respectively. The cell viability of cell lines decreased with 267 increasing drug concentration (Fig. 6A, 6C). Knockdown of Keap1 promoted cell viability and resisted 268 A549 and H1299 cells to cisplatin and paclitaxel, while overexpression of Keap1 showed the opposite result 269 (Fig. 6A, 6C). Cell viability was assessed using the CCK8 assay. In addition, the IC50 of cisplatin and 270 paclitaxel were reduced in both the cell lines over expressed Keap1 compared to the control cells, which 271 could be reversed by keap1 knockdown (Fig. 6B, 6D). These results indicated that Keap1 may confer 272 chemotherapy sensitivity in lung cancer cells.

\section{Keap1 knockdown promoted Nrf2 nucleus translocation.}

Keap1 is a major negative regulator of Nrf2, which has been shown to inhibit the proliferation and 275 cisplatin resistance of tumor cells. We therefore hypothesized that when the expression level of Keap1 was 276 abnormal, the expression level of Nrf2 would change accordingly. We then compared the protein expression 277 levels of total Nrf2 (T-Nrf2), nucleus Nrf2 (N-nrf2), and Heme Oxygenase-1 (HO-1) regulated by Nrf2 in 278 Keap1 KD or OE cell lines, respectively, and found that both T-Nrf2 and N-Nrf2 were reduced upon Keap1 279 overexpression, and downstream HO-1 was also reduced, while knockdown of Keap1 showing the opposite 280 results (Fig. 7A, 7B). The results were further confirmed by treating tumor cells with a certain concentration 281 of H2O2 (A549 $200 \mu \mathrm{M}, \mathrm{H} 1299100 \mu \mathrm{M}$ ) for 30 min (Fig. 7C, 7D). 


\section{Discussion}

283 The antioxidant stress system protects normal cells from reactive oxygen species and also creates

284 favorable conditions for the survival of tumor cells. The tumor microenvironment (TME) is a region where

285 tumor inflammation, hypoxia and oxidative stress coexist. and is a well-recognized actor in the development

286 of cancer and adaptation of tumor cells to therapy ${ }^{22,23}$. The Keap1-Nrf2 signaling pathway is the most

287 important anti-oxidative stress pathway in vivo, and its aberrant activation has been found in various

288 tumors, such as esophageal cancer and colon cancer $^{24,25}$. At present, it is generally believed that aberrant

289 activation of $\mathrm{Nrf} 2$ promotes radioresistance and chemoresistance of various cancers ${ }^{26}$. However, as the

290 master inhibitor of Nrf2, the role of Keap1 in tumors is controversial. On the one hand, Keap1 plays a role

291 similar to that of oncogenes, and on the other hand, Keap1 abnormalities promote the biological malignant

292 effects of tumor cells and are associated with poor prognosis in solid tumors ${ }^{27,28}$.

293 In the present study, we utilized immunohistochemistry analysis to detect the expression of Keap1 in

29437 surgically resected lung adenocarcinoma and adjacent tissues. We identified that Keap1 expression in

295 cancerous tissues was statistically lower compared to adjacent tissues. Keap1 expression was inversely

296 correlated with the clinical stage and lymph node metastasis and early-stage tumors (I) without lymph node

297 metastasis had higher levels of Keap1 expression compared with late-stage tumors (II and III). But the

298 expression of Keap1 was independent of age, gender, smoking history, tumor location, degree of

299 differentiation and tumor maximum diameter. Our results are consistent with previous research ${ }^{29}$. These

300 findings suggest that Keap1 may be involved in tumor progression, which could provide a new target for

301 intervention in the treatment of LUAD.

302 Keap1 is important in protection of oxidative stress. Recent studies have shown that Keap1 affects

303 tumor malignant behaviors. Overexpression of Keap1 in glioma cells decreased their migratory and

304 proliferative behaviors while knockdown of Keap1 increased this abrogating behavior ${ }^{30}$. These phenomena

305 are consistent with those observed in breast cancer cells ${ }^{31}$.Animal experiments further indicated that Keap1

306 R320Q somatic mutation could accelerate tumor growth in nude mouse ${ }^{32}$. In this study, we observed that

307 high levels of Keap1 expression significantly correlated with lower proliferation

Peer) reviewing PDF | (2020:12:56716:1:1:NEW 31 May 2021) 
308 and migration of LUAD cells and Keap1 knockdown facilitated EMT of tumor cells. We hypothesized that

309 this may be related to the effect of Nrf2 expression. It has been reported that Nrf2 nuclear translocation

310 could activate Notch1/Snail signaling pathway, which accelerated EMT and metastasis of tumor cells ${ }^{33}$. In

311 addition, PDCD4 inhibits the EMT pathway in tumors by increasing Keap1 expression and decreasing the

312 level and activity of $\mathrm{Nrf}^{34}$, our study also found that Keap1 knockdown promoted Nrf2 nuclear

313 translocation. However, the specific pathways by which Keap1 affects metastasis of LUAD cells require

314 further mechanistic studies. These observations suggest that Keap1 protects lung cancer proliferation and 315 metastasis and may serve as a biomarker for LUAD.

316 Chemotherapy resistance effectively blocks lung cancer treatment efficacy. It has been reported that

317 Nrf2 contributed to chemotherapy resistance of tumors. LncRNA TUG1 increases cisplatin resistance in

318 prostate cancer and esophageal cancer by the Nrf2 signal axis ${ }^{35,36}$. Nrf2 knockdown enhances sensitivity to

319 cytarabine and daunorubicin in acute myelogenous leukemia cell lines and inhibits tumor growth in a mouse

320

321

322

323

324

325

326

327

328

329

330

331

332

\section{3}

model of myelodysplastic syndrome ${ }^{37,38 .}$ As a master inhibitor of Nrf2, Keap1 is inextricably linked to

chemoresistance. In the present study, we treated aberrant Keap1 expression in A549 and H1299 cell lines with different concentrations of chemotherapy drugs and founded that overexpression of Keap1 may conferred the chemotherapy sensitivity in tumors.

In conclusion, this study investigated the effect of Keap1 on lung adenocarcinoma growth and drug resistance more systematically through clinical and cellular experiments. The results showed that Keap1 was lowly expressed in LUAD. The high expression level of Keap1 protein inhibited the growth and metastasis of LUAD cells and enhanced the sensitivity to chemotherapy, suggesting an anti-tumor effect of Keap1. It is speculated that the possible reason for this effect is related to the action of Keap1 on Nrf2. Overexpression of Keap1 promotes Nrf2 degradation and reduces Nrf2 nuclear translocation, which affects the subsequent anti-oxidative stress effect and disrupts the balance of TME. Ultimately, it inhibits tumor cell growth and metastasis, decreases the expression of antitumor drug efflux-related proteins and glutathione levels, and enhances cellular sensitivity to chemotherapy sensitivity.

\section{Conclusions}

Peer) reviewing PDF | (2020:12:56716:1:1:NEW 31 May 2021) 
334 Our conclusion confirmed the involvement of Keap1 in LUAD progression and drug sensitivity. 335 Keap1 is expected to serve as a potential tumor marker to guide the staging and treatment of lung cancer. 336 However, there are still limitations: first, the relatively small sample size may lead to selection bias, and 337 the lack of clinical response of patients to chemotherapy may weaken the validity of the results. Second, 338 the effect of Keap1 on LUAD is limited to the cellular phenotype, and further investigation of its molecular 339 mechanisms is essential.

\section{Acknowledgements}

341 We wish to thank Mr. Lun Zhu for his assistance in collecting clinical specimens. 


\section{References}

343 1. Bray F, Ferlay J, Soerjomataram I, Siegel RL, Torre LA, Jemal A. Global cancer statistics 2018: 344 GLOBOCAN estimates of incidence and mortality worldwide for 36 cancers in 185 countries. CA $345 \quad$ Cancer J Clin. 2018;68(6):394-424.

346 2. Cao M, Chen W. Epidemiology of lung cancer in China. Thorac Cancer. 2019;10(1):3-7.

347 3. Dhanoa BS, Cogliati T, Satish AG, Bruford EA, Friedman JS. Update on the Kelch-like (KLHL) $348 \quad$ gene family. Hum Genomics. 2013;7(1):13.

349 4. Ogura T, Tong KI, Mio K, Maruyama Y, Kurokawa H, Sato C, Yamamoto M. Keap1 is a forked350 stem dimer structure with two large spheres enclosing the intervening, double glycine repeat, and 351 C-terminal domains. Proc Natl Acad Sci U S A. 2010;107(7):2842-7.

352

5. Canning P, Sorrell FJ, Bullock AN. Structural basis of Keap1 interactions with Nrf2. Free Radic Biol Med. 2015;88(Pt B):101-7

354

6. Luo Y, Eggler AL, Liu D, Liu G, Mesecar AD, van Breemen RB. Sites of alkylation of human Keap1 by natural chemoprevention agents. J Am Soc Mass Spectrom. 2007;18(12):2226-32.

356

7. Tsuchiya M, Misaka R, Nitta K, Tsuchiya K. Mafs and their biological roles. World J Diabetes.

357 $2015 ; 6(1): 175-83$.

358 8. Bellezza I, Giambanco I, Minelli A, Donato R. Nrf2-Keap1 signaling in oxidative and reductive 359 stress. Biochim Biophys Acta Mol Cell Res. 2018;1865(5):721-33.

360 .9. Schulze K, Imbeaud S, Letouzé E, Alexandrov LB, Calderaro J, Rebouissou S, Couchy G, Meiller 361 C. Exome sequencing of hepatocellular carcinomas identifies new mutational signatures and potential therapeutic targets. Nat Genet. 2015;47(5):505-11.

10. Chu XY, Li ZJ, Zheng ZW, Tao YL, Zou FX, Yang XF. KEAP1/NRF2 signaling pathway mutations in cervical cancer. Eur Rev Med Pharmacol Sci. 2018;22(14):4458-66.

365 11. Matthew Meyerson. Comprehensive genomic characterization of squamous cell lung cancers. Nature. 2012;489(7417):519-25. 
367 12. Frank R, Scheffler M, Merkelbach-Bruse S, Ihle MA, Kron A, Rauer M, Ueckeroth F, König K. 368 Clinical and Pathological Characteristics of KEAP1- and NFE2L2-Mutated Non-Small Cell Lung 369 Carcinoma (NSCLC). Clin Cancer Res. 2018;24(13):3087-96.

370 13. Ahtikoski AM, Kangas J, Salonen R, Puistola U, Karihtala P. Cytoplasmic Keap1 Expression Is 371 Associated With Poor Prognosis in Endometrial Cancer. Anticancer Res. 2019;39(2):585-90.

372 14. Isohookana J, Haapasaari KM, Soini Y, Karihtala P. Keap1 expression has independent prognostic 373 value in pancreatic adenocarcinomas. Diagn Pathol. 2015;10:28.

374 15. Zhang L, Yang WP, Wu LY, Zhu X, Wei CY. Expression and clinical significance of Kelch-like 375

16. Zhang J, Wang X, Wu W, Dang H, Wang B. Expression of the Nrf2 and Keap1 proteins and their clinical significance in osteosarcoma. Biochem Biophys Res Commun. 2016;473(1):42-6.

378 17. Detterbeck FC, Boffa DJ, Kim AW, Tanoue LT. The Eighth Edition Lung Cancer Stage 379 Classification. Chest. 2017;151(1):193-203.

380

18. Fromowitz FB, Viola MV, Chao S, Oravez S, Mishriki Y, Finkel G, Grimson R, Lundy J. ras p21 expression in the progression of breast cancer. Hum Pathol. 1987;18(12):1268-75.

382

19. Wang Y, Wang H, Pan T, Li L, Li J, Yang H. STIM1 silencing inhibits the migration and invasion 383 of A549 cells. Mol Med Rep. 2017;16(3):3283-9.

20. Zhang W, Shi H, Chen C, Ren K, Xu Y, Liu X, He L. Curcumin enhances cisplatin sensitivity of

21. Sun H, Zhou X, Bao Y Xiong G, Cui Y, Zhou H. Involvement of miR-4262 in paclitaxel resistance 388

22. Hinshaw DC, Shevde LA. The Tumor Microenvironment Innately Modulates Cancer Progression. Cancer research 2019, 79(18):4557-4566.

391 23. Paardekooper LM, Vos W, van den Bogaart G. Oxygen in the tumor microenvironment: effects on dendritic cell function. Oncotarget, 2019, 10(8):883-896. 
393 24. Zhang J, Jiao Q, Kong L, Yu J, Fang A, Li M, Yu J. Nrf2 and Keap1 abnormalities in esophageal 394 squamous cell carcinoma and association with the effect of chemoradiotherapy. Thorac Cancer. $395 \quad 2018 ; 9(6): 726-35$.

396 25. Sadeghi MR, Jeddi F, Soozangar N, Somi MH, Samadi N. The role of Nrf2-Keap1 axis in colorectal 397 cancer, progression, and chemoresistance. Tumour Biol. 2017;39(6):1010428317705510.

398 26. Leinonen HM, Kansanen E, Pölönen P, Heinäniemi M, Levonen AL. Dysregulation of the Keap1399 Nrf2 pathway in cancer. Biochem Soc Trans. 2015;43(4):645-9.

400 27. Fabrizio FP, Sparaneo A, Centra F, Trombetta D, Storlazzi CT, Graziano P, Maiello E, Fazio VM. 401 Methylation Density Pattern of KEAP1 Gene in Lung Cancer Cell Lines Detected by Quantitative 402 Methylation Specific PCR and Pyrosequencing. Int J Mol Sci. 2019;20(11).

403 28. Fabrizio FP, Costantini M, Copetti M, la Torre A, Sparaneo A, Fontana A, Poeta L, Gallucci M. 404 Keap1/Nrf2 pathway in kidney cancer: frequent methylation of KEAP1 gene promoter in clear renal cell carcinoma. Oncotarget. 2017;8(7):11187-98.

29. Chien MH, Lee WJ, Hsieh FK, Li CF, Cheng TY, Wang MY, Chen JS, Chow JM, Jan YH, Hsiao 407 M. Keap1-Nrf2 Interaction Suppresses Cell Motility in Lung Adenocarcinomas by Targeting the S100P Protein. Clin Cancer Res. 2015;21(20):4719-32.

409

30. Fan Z, Wirth AK, Chen D, Wruck CJ, Rauh M, Buchfelder M, Savaskan N. Nrf2-Keap1 pathway promotes cell proliferation and diminishes ferroptosis. Oncogenesis. 2017;6(8):e371.

411 31. Zhang HS, Zhang ZG, Du GY, Sun HL, Liu HY, Zhou Z, Gou XM, Wu XH, Yu XY. Huang YH,. 412 Nrf2 promotes breast cancer cell migration via up-regulation of G6PD/HIF-1 $\alpha /$ Notch1 axis. J Cell $413 \quad$ Mol Med. 2019;23(5):3451-63.

414 32. Gong M, Li Y, Ye X, Zhang L, Wang Z, Xu X, Shen Y, Zheng C. Loss-of-function mutations in 415 KEAP1 drive lung cancer progression via KEAP1/NRF2 pathway activation. Cell Commun Signal. $416 \quad 2020 ; 18(1): 98$.

417 33. Han M, Li N, Li F , Wang H, Ma L. MiR-27b-3p exerts tumor suppressor effects in esophageal 418 squamous cell carcinoma by targeting Nrf2. Human cell 2020, 33(3):641-651. 
419 34. Hwang SK, Jeong YJ, Chang YC. PDCD4 inhibits lung tumorigenesis by the suppressing p62-Nrf2 420 signaling pathway and upregulating Keap1 expression. American journal of cancer research 2020, $421 \quad 10(2): 424-439$.

422 35. Yang G, Yin H, Lin F, Gao S, Zhan K, Tong H, Tang X, Pan Q, Gou X. Long noncoding RNA 423 TUG1 regulates prostate cancer cell proliferation, invasion and migration via the Nrf2 signaling 424 axis. Pathol Res Pract. 2020;216(4):152851.

425 36. Zhang Z, Xiong R, Li C, Xu M, Guo M. LncRNA TUG1 promotes cisplatin resistance in 426 esophageal squamous cell carcinoma cells by regulating Nrf2. Acta Biochim Biophys Sin 427 (Shanghai). 2019;51(8):826-33.

428 37. Karathedath S, Rajamani BM, Musheer Aalam SM, Abraham A, Varatharajan S, Krishnamurthy 429 P. Role of NF-E2 related factor $2(\mathrm{Nrf} 2)$ on chemotherapy resistance in acute myeloid leukemia 430 (AML) and the effect of pharmacological inhibition of Nrf2. PLoS One. 2017;12(5):e0177227.

431 38. Lin P, Ren Y, Yan X, Luo Y, Zhang H, Kesarwani M, Bu J, Zhan D, Zhou Y, Tang Y, Zhu S. The 432 high NRF2 expression confers chemotherapy resistance partly through up-regulated DUSP1 in myelodysplastic syndromes. Haematologica. 2019;104(3):485-96. 


\section{Table 1 (on next page)}

The primary antibody used in western blot 
Table1 The primary antibody used in western blot

\begin{tabular}{lll}
\hline \multicolumn{1}{c}{ Name } & \multicolumn{1}{c}{ Product number } & \multicolumn{1}{c}{ Company } \\
\hline Keap1 & ab227828 & Abcam \\
Nrf2 & ab62352 & Abcam \\
E-cadherin & $\# 3195$ & Cell Signaling \\
N-cadherin & ab76011 & Abcam \\
Vimentin & $10366-1-A P$ & Proteintech \\
Snail & ab216347 & Abcam \\
$\beta$-actin & $20536-1-A P$ & Proteintech \\
LaminB & ab16048 & Abcam \\
\hline
\end{tabular}

1 
Table 2 (on next page)

Correlation of Keap1 with the occurrence of LUAD 
1

2

3

4

5

6

\begin{tabular}{lccccc}
\multicolumn{2}{l}{ Table 2} & \multicolumn{2}{c}{ Correlation of Keap1 with the occurrence of LUAD } & $P$ \\
\hline \multirow{2}{*}{ Group } & $\mathrm{n}$ & \multicolumn{2}{c}{ Keap1 } & 0.019 \\
\cline { 3 - 4 } & 37 & Low expression(\%) & High expression(\%) & \\
LUAD & $33(62.2)$ & $14(37.8)$ & \\
para-cancerous & 37 & $12(32.4)$ & $25(67.6)$ & \\
\hline
\end{tabular}


Table 3(on next page)

Baseline characteristics of LUAD and the correlation with Keap1 expression 
Table 3 Baseline characteristics of LUAD and the correlation with Keap1 expression

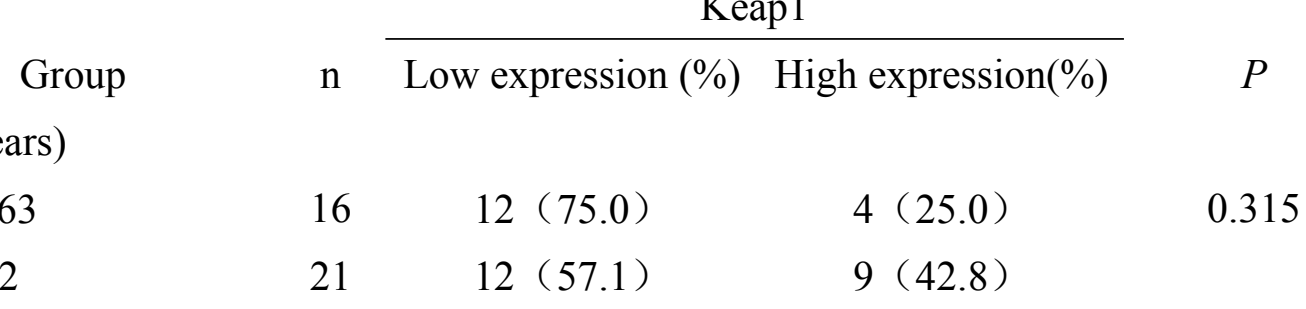

Gender

$\begin{array}{lllll}\text { Male } & 19 & 14(73.7) & 5(26.3) & 0.313 \\ \text { Female } & 18 & 10(55.6) & 8(44.4) & \end{array}$

Smoking

$\begin{array}{lllcl}\text { Yes } & 12 & 10(83.3) & 2(16.7) & 0.149 \\ \text { No } & 25 & 14(56.0) & 11(44.0) & \end{array}$

Tumour site

$\begin{array}{lllll}\text { Left } & 15 & 11(73.3) & 4(26.7) & 0.491 \\ \text { Right } & 22 & 13(59.1) & 9(40.9) & \end{array}$

Differentiation

$\begin{array}{lcccc}\text { Low } & 5 & 4(80.0) & 1(20.0) & 0.634 \\ \text { Moderate } & 19 & 13(68.4) & 6(31.6) & \\ \text { High } & 13 & 7(53.8) & 6(46.2) & \end{array}$

Stage

$\begin{array}{lllll}\text { I } & 15 & 6(40.0) & 9(60.0) & \mathbf{0 . 0 3 0} \\ \text { II } & 12 & 9(75.0) & 3(25.0) & \\ \text { III } & 10 & 9(90.0) & 1(10.0)\end{array}$

Lymph node metastasis

$\begin{array}{llccc}\text { Yes } & 16 & 14(87.5) & 2(12.5) & \mathbf{0 . 0 1 7} \\ \text { No } & 21 & 10(47.6) & 11(52.4) & \end{array}$

Tumor max diameter

\begin{tabular}{lllll}
$>3.56 \mathrm{~cm}$ & 16 & $12(75.0)$ & $4(25.0)$ & 0.315 \\
$\leq 3.56 \mathrm{~cm}$ & 21 & $12(57.1)$ & $9(42.9)$ & \\
\hline
\end{tabular}




\section{Figure 1}

Keap1 was low expressed in LUAD tissue and cell lines.

Figure1 A-B Representative immunohistochemical images with staining for Keap1 in different clinical stages of LUAD and matched non-cancerous samples. Photomicrographs were captured under x400 magnification. Scale bar 20 um. (A) Low Keap1 expression in LUAD (Stage III, Left; Stage II, Right); (B) High Keap1 expression in LUAD (Stage I,Left) and normal (Right). (C) Keap1 expression was tested and compared between human bronchial epithelial cell line HBE and lung cancer cell lines via Western blot analysis, $\beta$-actin protein was served as an internal control. (D) Quantitative analysis of Keap1 protein in different cell lines was shown. (E) Expression of keap1 in different lung cancer cell lines and human bronchial epithelial cells line HBE was compared by RT-qPCR. GAPDH was used as a reference for RNA. Statistical significance was tested by unpaired t test. Values were given as mean $\pm S D *$ $\mathrm{P}<0.05, * * \mathrm{P}<0.01 * * * \mathrm{P}<0.001$ compared to the control group(HBE). 


\section{Low expression}

A Stagellif:

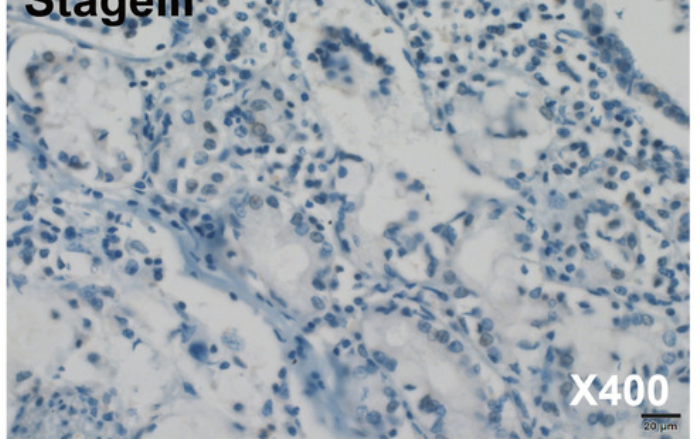

High expression

B

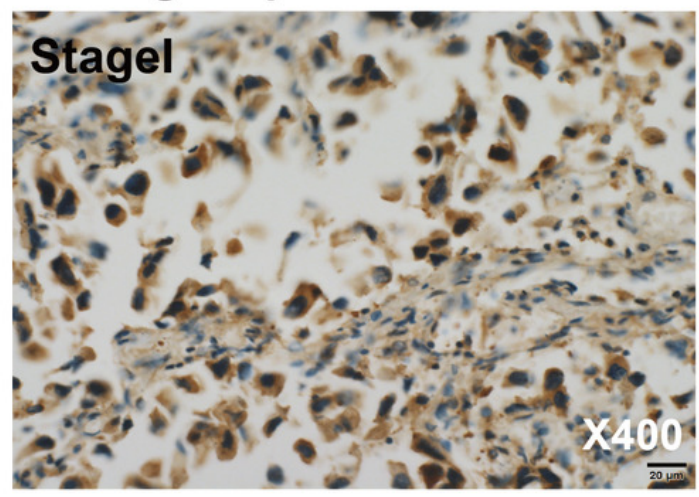

Low expression

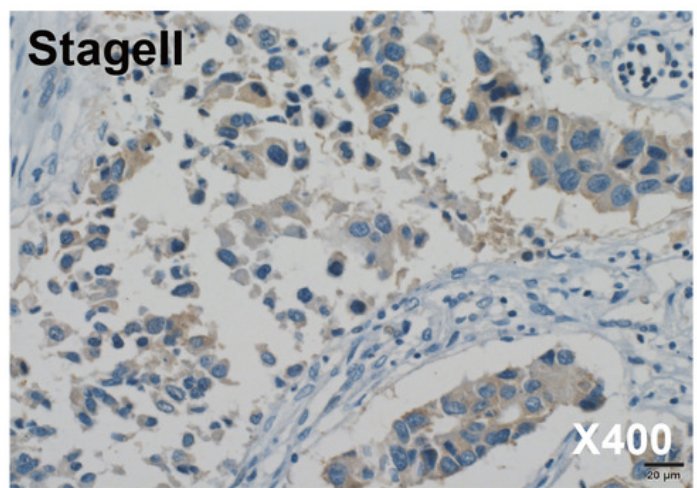

High expression

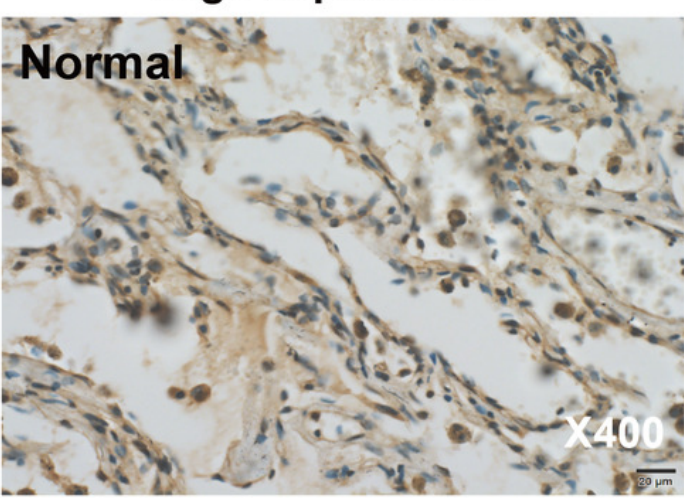

C

HBE A549 H2126 H460 H1299

Keap1

$42 k d$

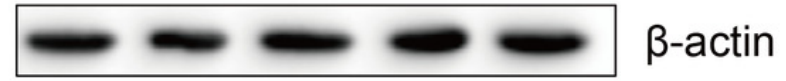

D

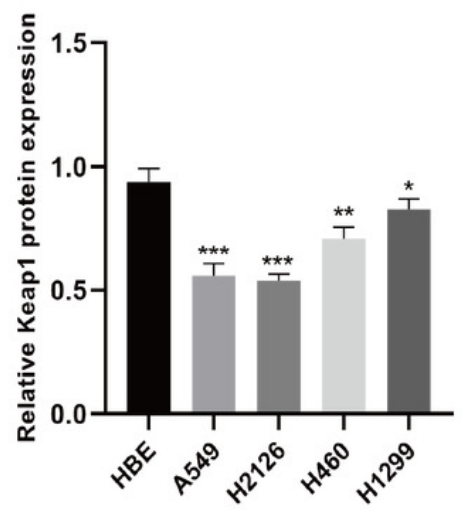

E

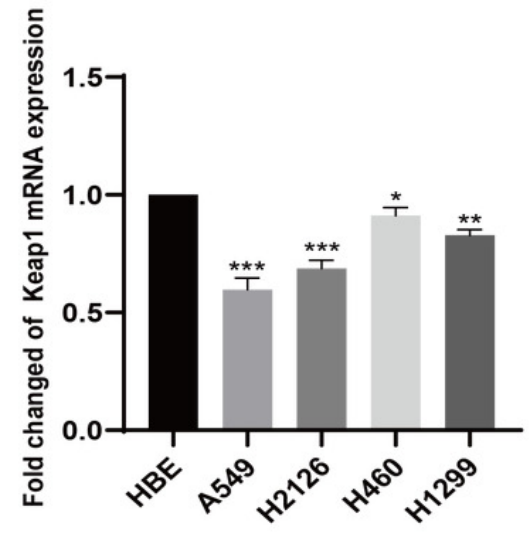




\section{Figure 2}

Keap1 protein and mRNA expression levels by western blot analysis and RT-qPCR.

Figure2 (A) Expression of Keap1 protein level in A549 and H1299 cell lines was performed by western blotting analysis. $\beta$-actin protein was served as an internal control. (B) Quantitative analysis of Keap1 protein in A549 and H1299 cell lines was shown. (C) Expression of Keap1 mRNA level in A549 and H1299 cell lines was examined by RT-qPCR. GAPDH was used as a reference for RNA. Statistical significance was tested by unpaired t test. Values were given as mean $\pm S D . * P<0.05, * * P<0.01, * * * P<0.001$ compared to the control group. 
A

$\frac{2}{\text { A549 }}$

$70 \mathrm{kd}$

$42 \mathrm{kd}$

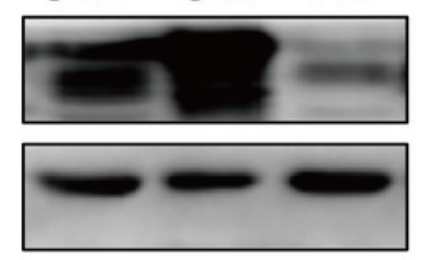

Keap1

$\beta$-actin

B

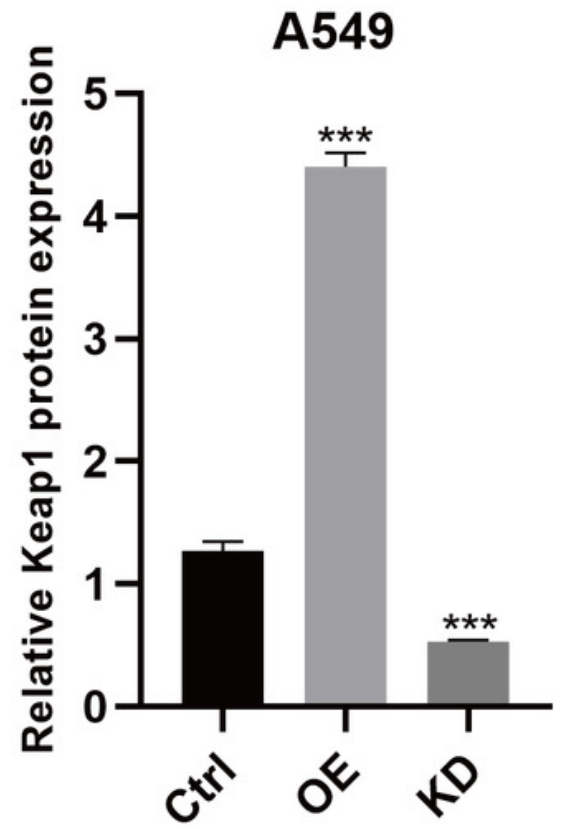

C

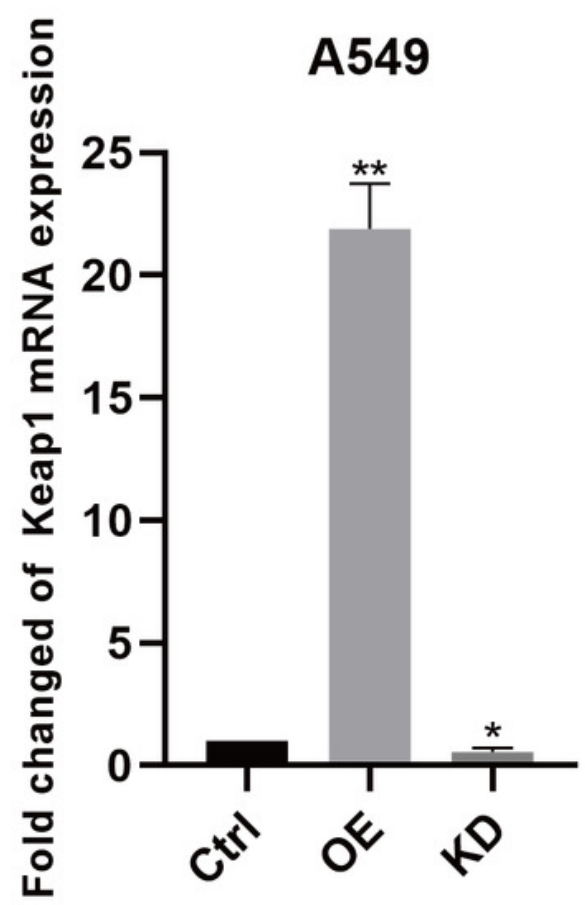

$\mathrm{H} 1299$

Ctrl OE KD

$70 \mathrm{kd}$

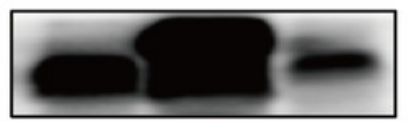

Keap1

$42 \mathrm{kd}$

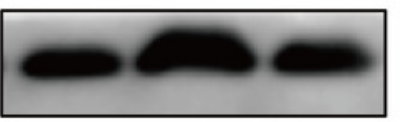

$\beta$-actin
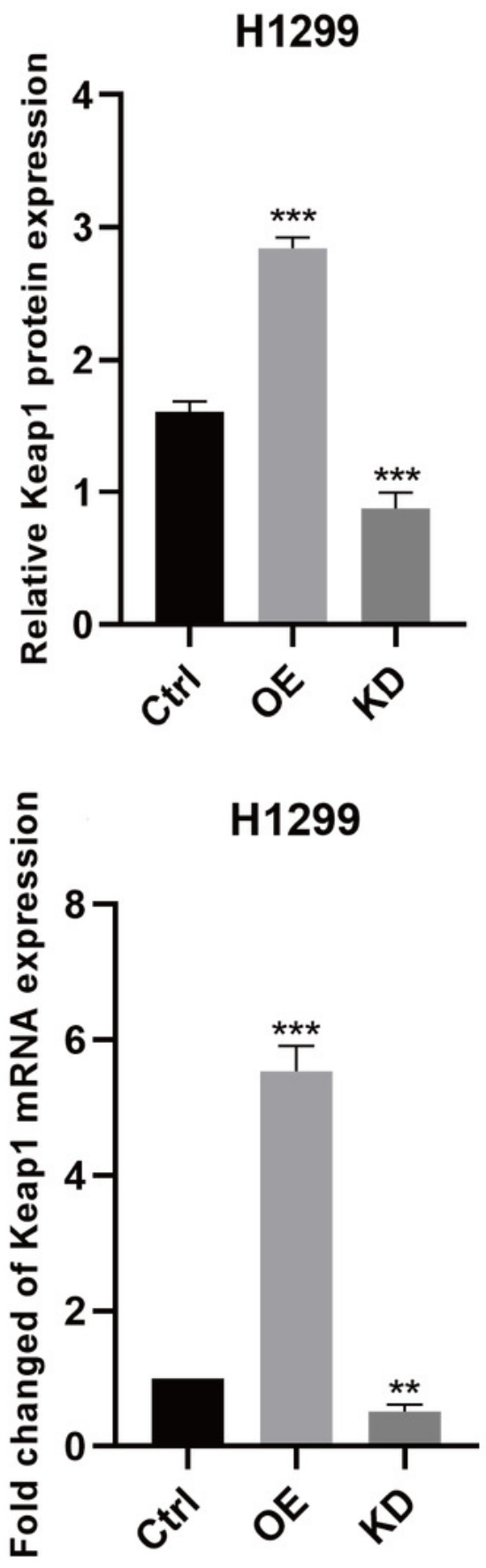


\section{Figure 3}

Keap1 knockdown facilitated A549 and H1299 cell lines proliferation.

Figure3 (A) Representative images of colony formation assay in A549 and H1299 cell lines expressing different levels of keap1 were shown. (B) Quantitative analysis of total colony numbers in A549 and H1299 cells was shown. (C) Cell proliferation abilities in A549 and H1299 cell lines expressing different levels of keap1 were analysed by cell growth curves. Statistical significance was tested by unpaired $t$ test. Values were given as mean $\pm S D . * * P<0.01, * * * P<0.001$ compared to the control group. 


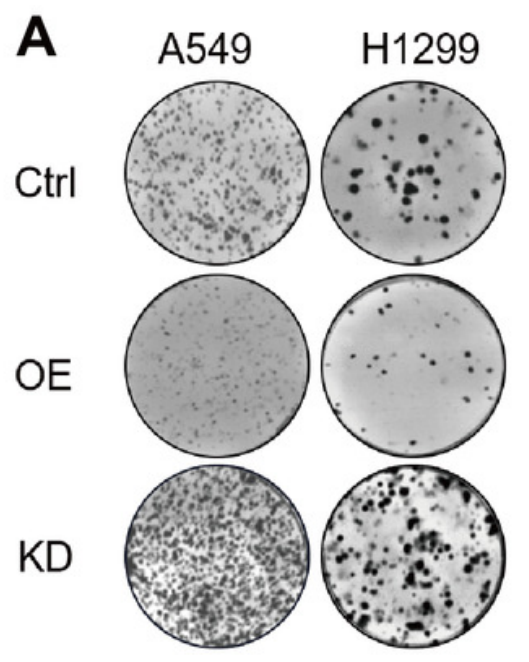

B
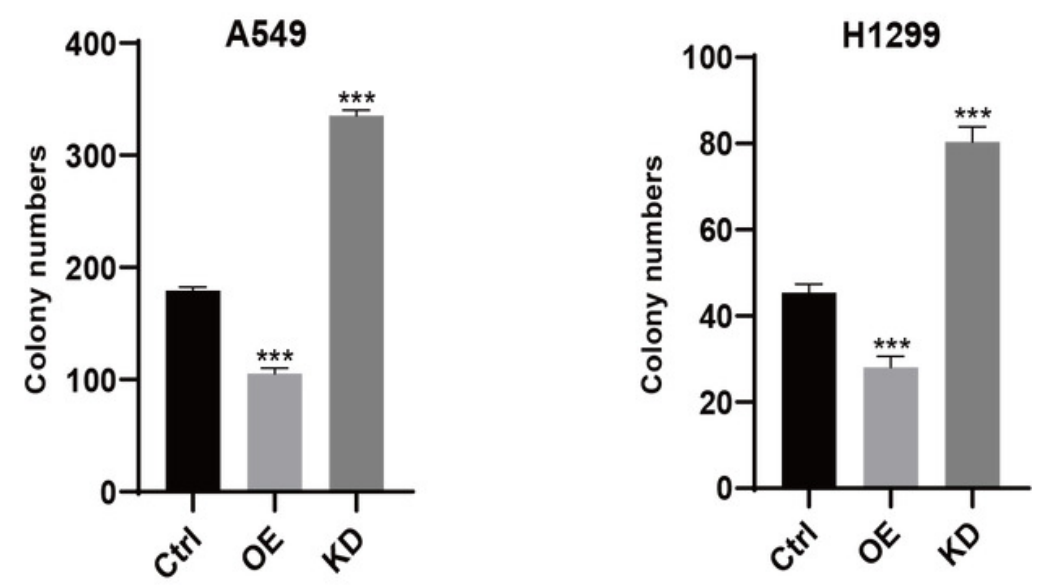

C
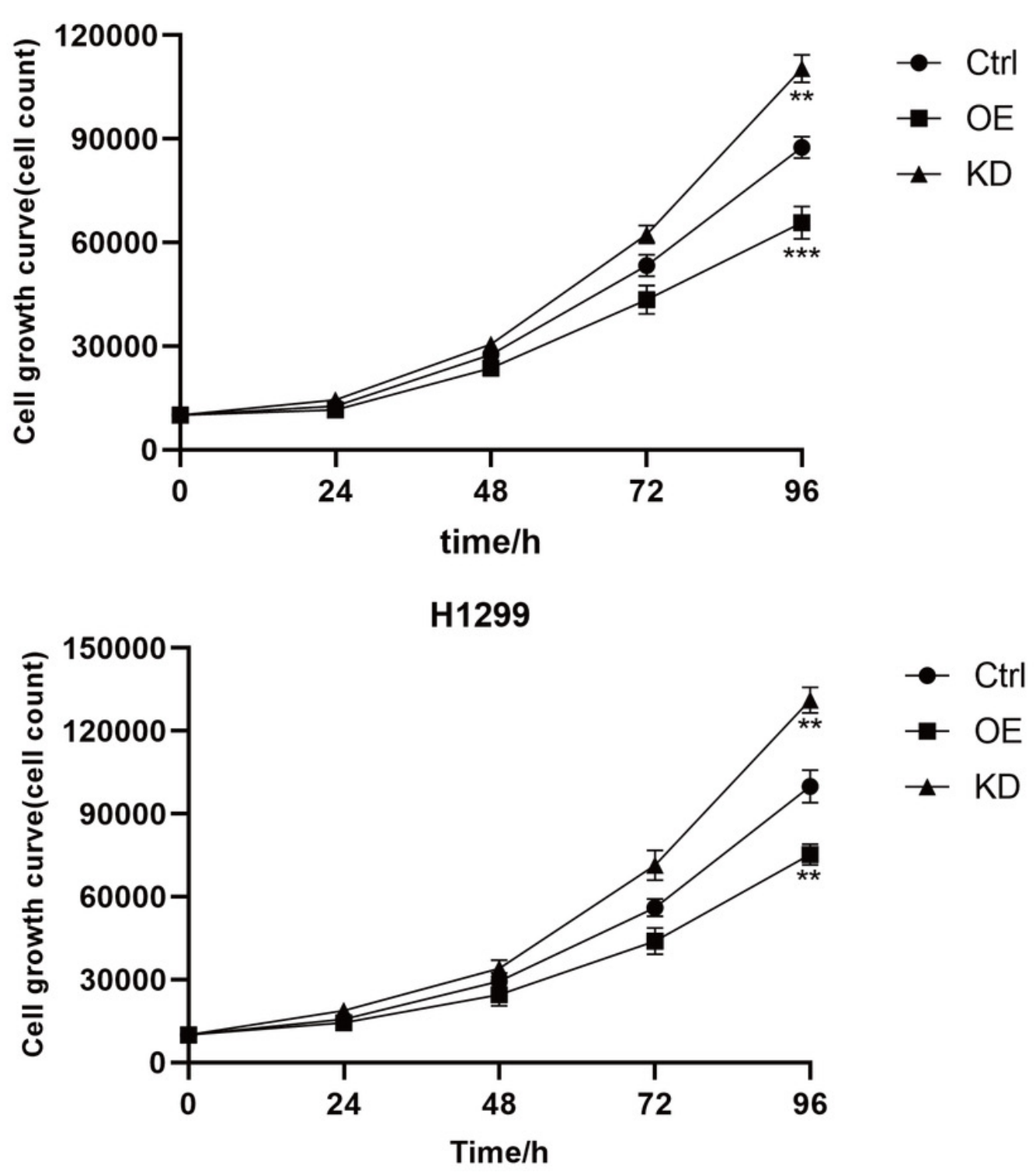


\section{Figure 4}

Keap1 knockdown promoted the migration and invasion in A549 and H1299 cell lines.

Figure4 (A) Wound scratch assay was performed with A549 and H1299 cell lines expressing different levels of keap1. (B) Quantitative analysis of wound scratch assay in A549 and H1299 cell lines expressing different levels of keap1. Images were captured at a certain time after wound was formed. The percentage of migration was assigned as 100\% when complete fusion occurred, and $0 \%$ at $\mathrm{t}=0 \mathrm{~h}$. Relative migratory rates were shown in the graphs. (C) Transwell invasion assay of A549 and H1299 cell lines expressing different levels of keap1. (D) Quantitative analysis of transwell invasion assay of A549 and H1299 cells was shown. Statistical significance was tested by unpaired $t$ test. Values were given as mean $\pm S D$. $* P<0.05, * * P<0.01 * * * P<0.001$ compared to the control group. 
A
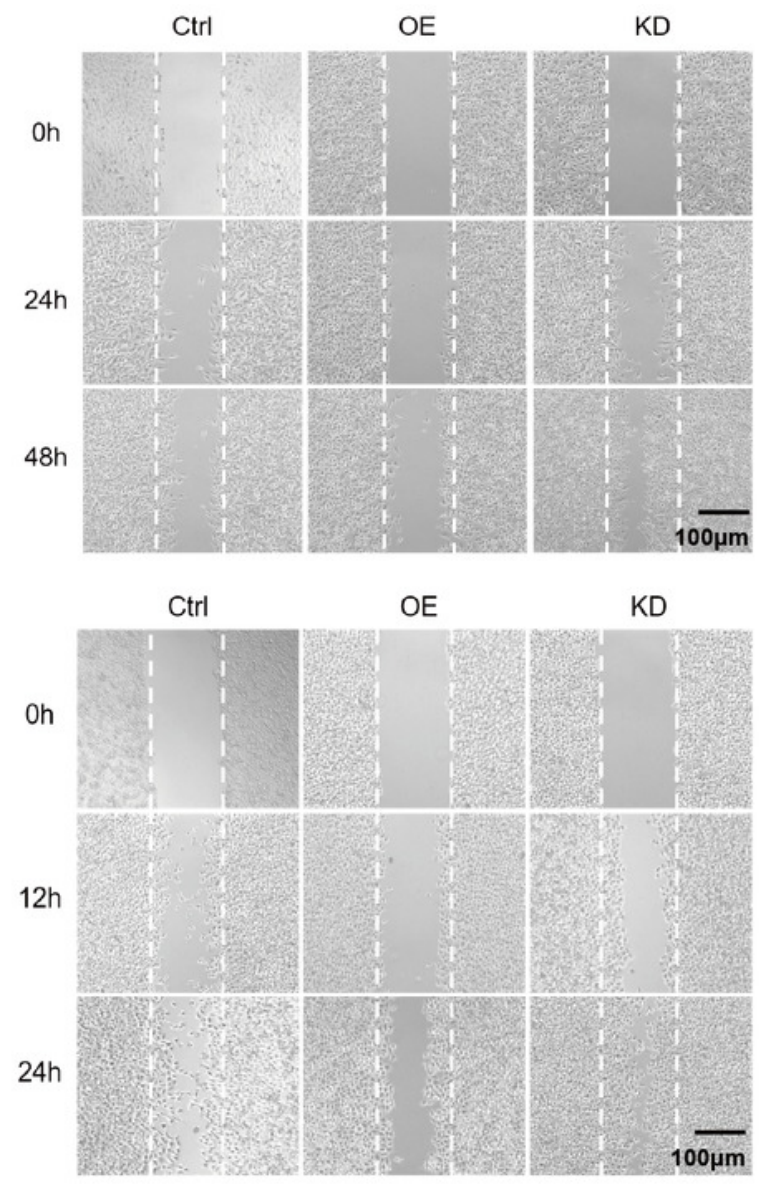

B
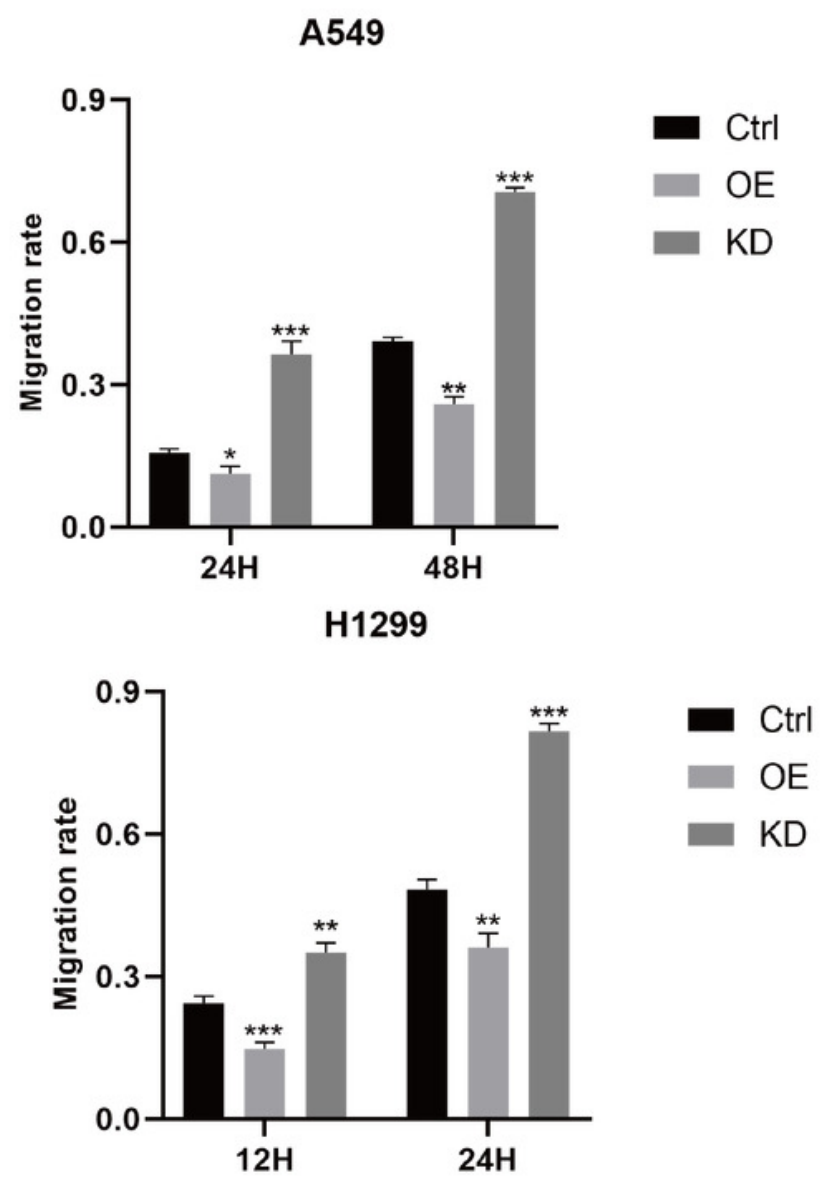

C

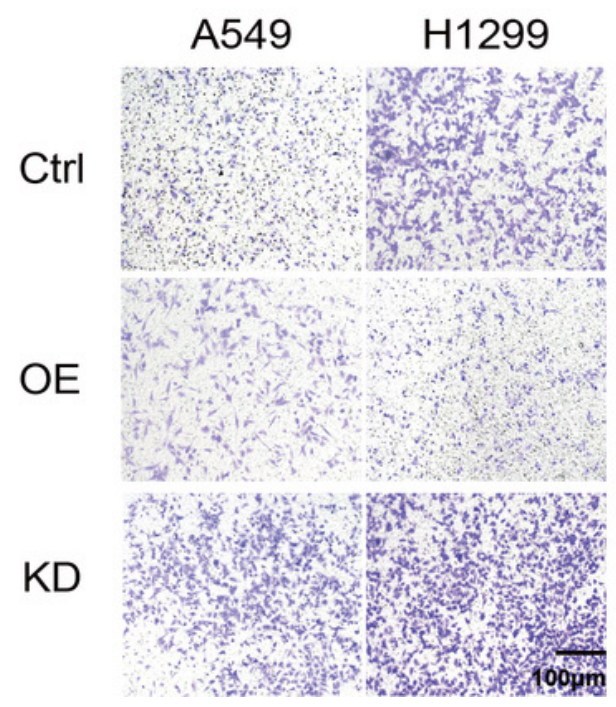

D

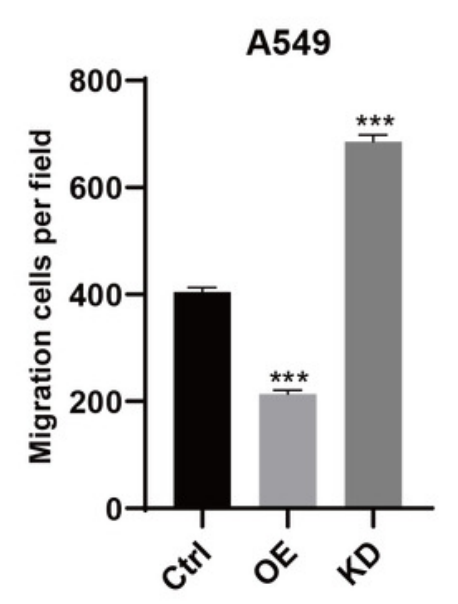

H1299

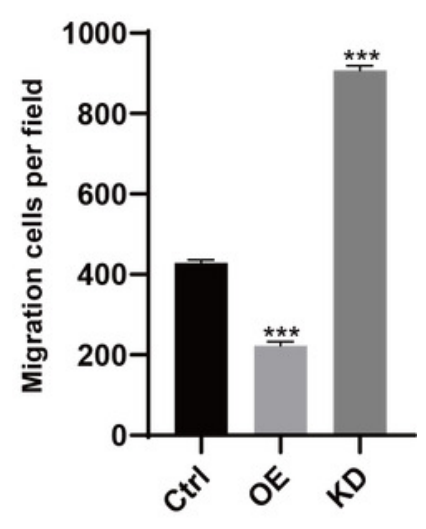




\section{Figure 5}

Keap1 knockdown facilitated EMT of A549 and H1299 cell lines.

Figure5 Keap1 knockdown facilitated EMT of A549 and H1299 cell lines. (A) Western blot analysis of epithelial markers E-cadherin, mesenchymal markers (N-cadherin, Vimentin) and Snail in A549 cells with different Keap1 expression levels. (B) Quantitative analysis of EMT protein in A549 cell line was shown. (C) Western blot analysis of epithelial markers Ecadherin, mesenchymal markers (N-cadherin, Vimentin) and Snail in H1299 cells with different Keap1 expression levels. (D) Quantitative analysis of EMT protein in H1299 cell line was shown. Statistical significance was tested by unpaired $t$ test. Values were given as mean \pm SD. $* P<0.05, * * P<0.01, * * * P<0.001$ compared to the control group. 
A

A549

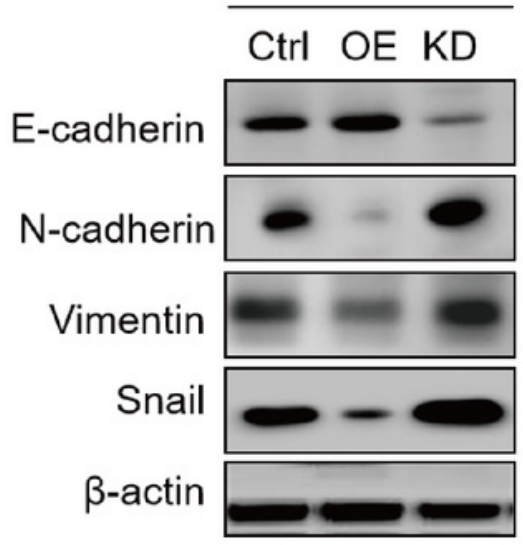

C

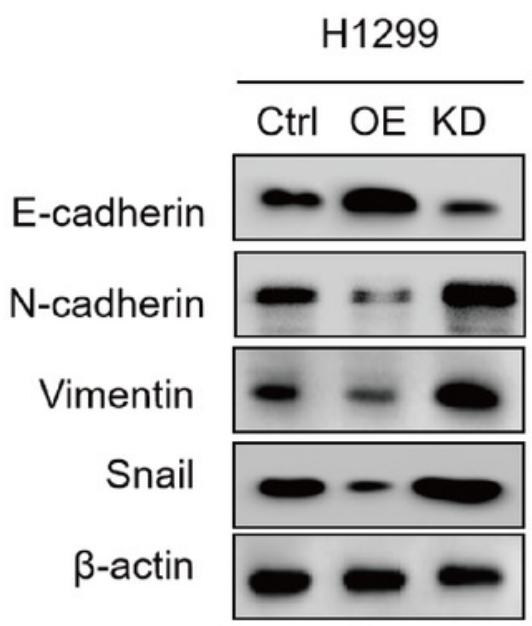

B

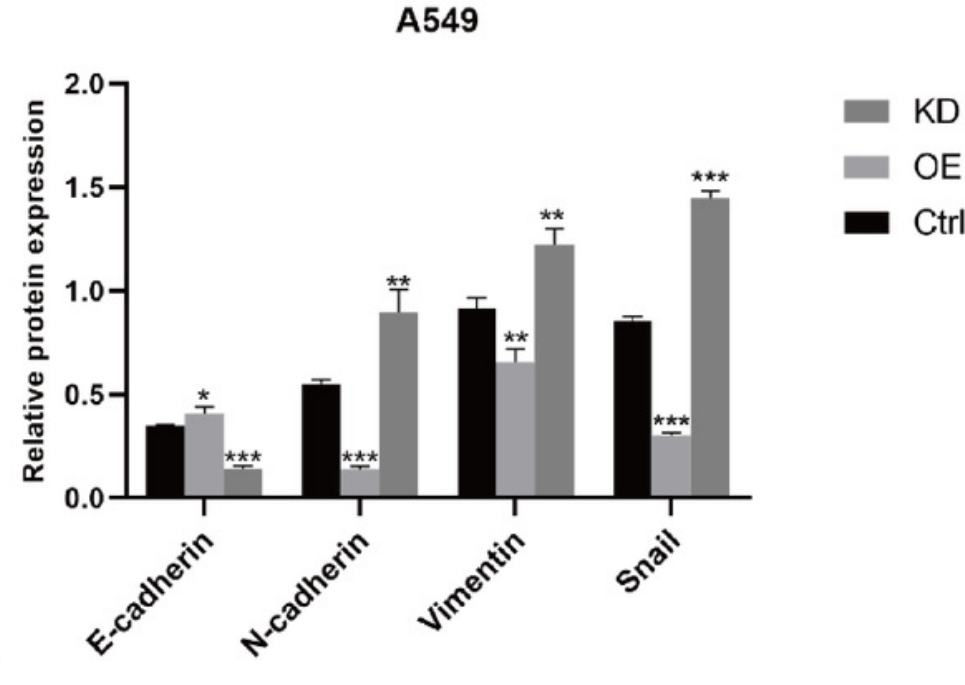

H1299

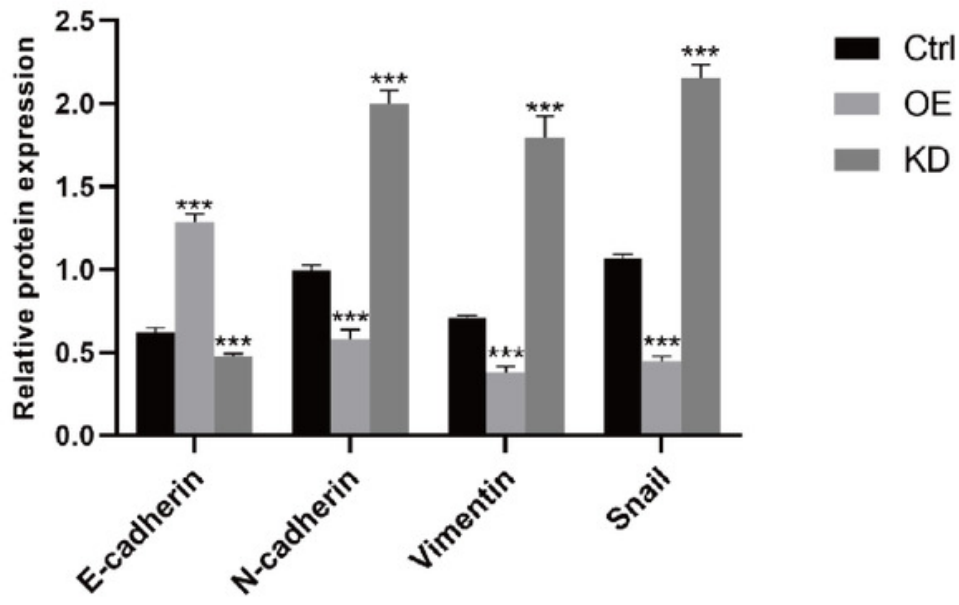




\section{Figure 6}

Keap1 knockdown confered the chemotherapy resistance in A549 and H1299 cells.

Figure6 Keap1 knockdown confered the chemotherapy resistance in A549 and H1299 cells.(A) A549 and H1299 cell liens expressing different levels of keap1 were treated with different concentrations of cisplatine, and the cell viability was determined by CCK8 assay. (B) IC50 of A549 and H1299 cell lines expressing different levels of keap1 to cisplatin. (C) A549 and H1299 cell liens expressing different levels of keap1 were treated with different concentrations of paclitaxel, and the cell viability was determined by CCK8 assay. (D) IC50 of A549 and H1299 cell lines expressing different levels of keap1 to paclitaxel. Statistical significance was tested by unpaired $t$ test. Values were given as mean $\pm S D$. * $P<0.05$,** $\mathrm{P}<0.01$ compared to the control group.

A

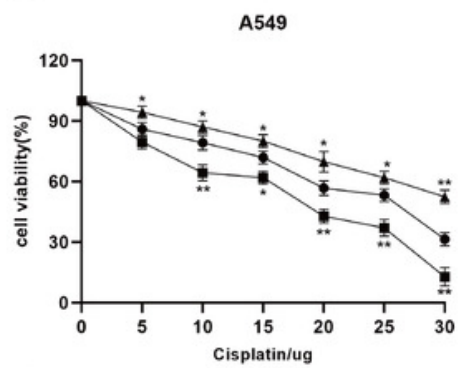

C

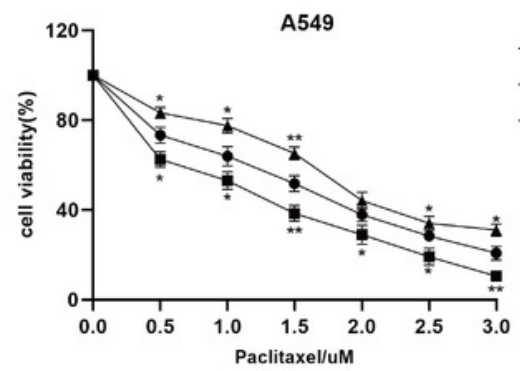

- $\mathrm{Ctr}$

$-\mathrm{Ctr}$
$-\mathrm{OE}$

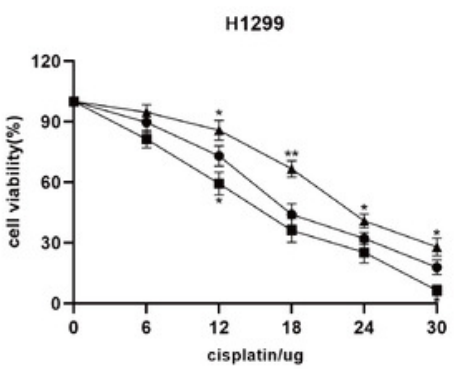

$-\mathrm{Ctrl}$
$-\mathrm{OE}$

$\star \mathrm{KD}$

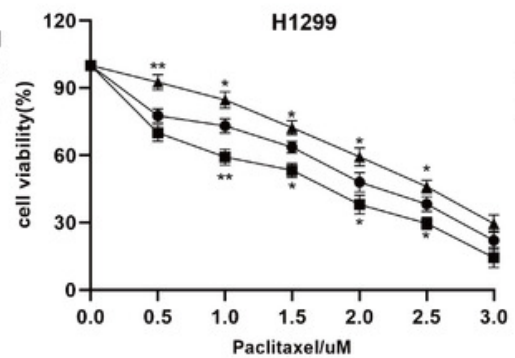

- Ctrl

$-\mathrm{OE}$

$\rightarrow \mathrm{KD}$
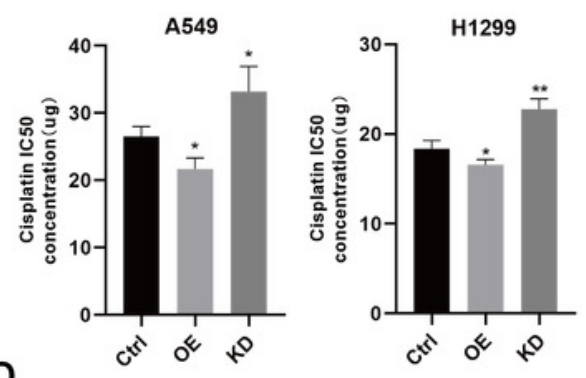

- Ctrl

$-\mathrm{OE}$
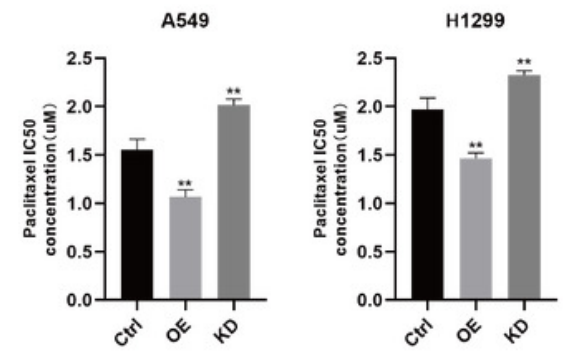


\section{Figure 7}

Keap1 knockdown promoted Nrf2 into the nucleus of tumor cells.

Figure7 Keap1 knockdown promoted Nrf2 into the nucleus of tumor cells. (A) Western blot analysis of total Nrf2 (T-Nrf2), HO-1 and nucleus Nrf2 (N-Nrf2) in two cells with different Keap1 expression levels. (B) Quantitative analysis of T-Nrf2, HO-1 and N-Nrf2 proteins in A549 and H1299 cell lines. (C) Western blot analysis of T-Nrf2, HO-1 and N-Nrf2 in cells with different Keap1 expression levels under oxidative stress (H2O2) conditions. (D) Quantitative analysis of T-Nrf2, HO-1 and N-Nrf2 proteins in cells with different Keap1 expression levels under oxidative stress ( $\mathrm{H} 2 \mathrm{O} 2$ ).Statistical significance was tested by unpaired $t$ test. Values were given as mean $\pm S D * P<0.05, * * P<0.01,{ }^{* * *} \mathrm{P}<0.001$ compared to the control group.
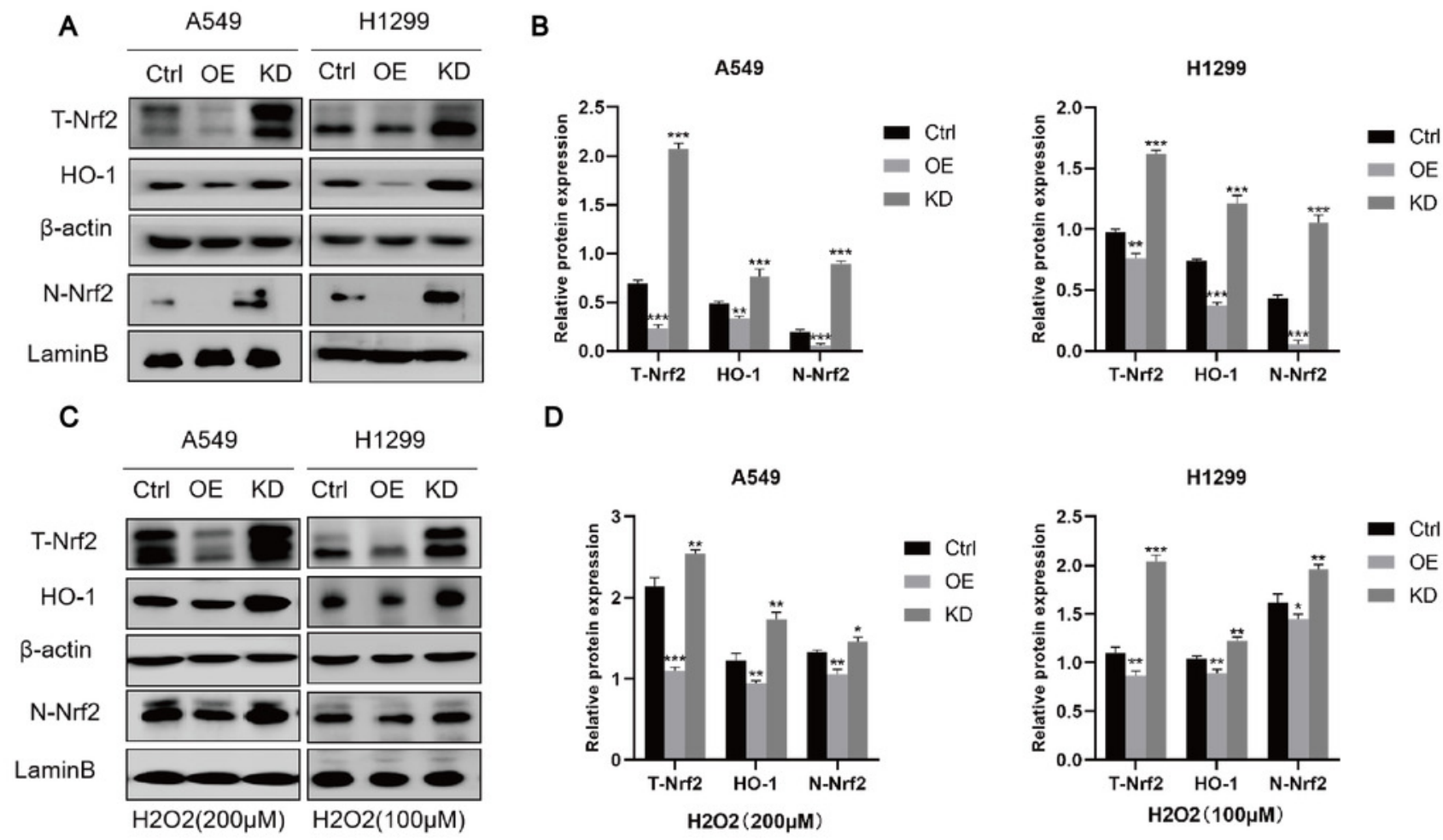

D
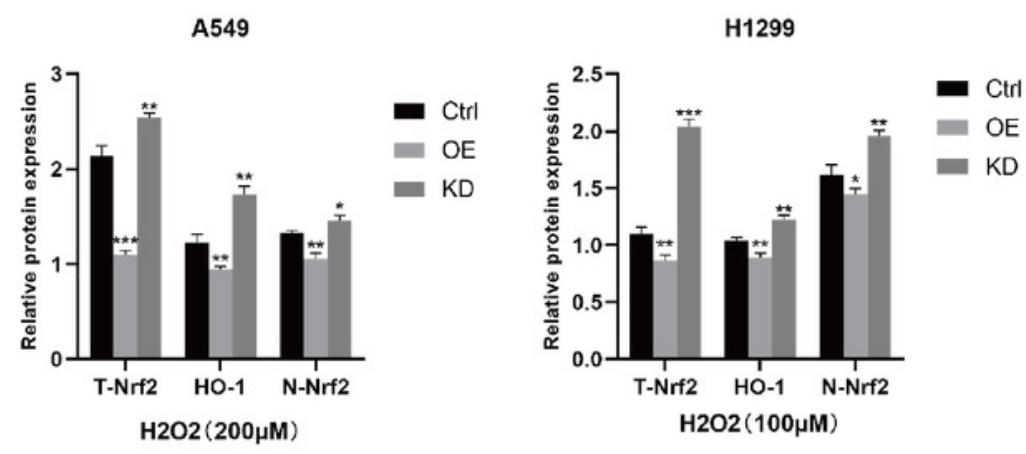